\title{
Model-based Prognostic of the Remaining Useful Life of Bearings Considering Model Parameter Uncertainty
}

Fatemeh Hosseinpour

Department of Mechanical Engineering, Sharif University of Technology, Tehran, Iran.

Department of Energy Department, Politecnico di Milano, Milan, Italy

E-mail:fatemeh.hosseinpour@mech.sharif.edu

Mehdi Behzad

Department of Mechanical Engineering, Sharif University of Technology, Tehran, Iran.

E-mail: m behzad@sharif.edu

Enrico Zio

Department of Energy, Politecnico di Milano, Milan, Italy.E-mail: enrico.zio@polimi.it

Centre de Recherche sur les Risques et les Crises (CRC), MINES ParisTech/PSL Université Paris, Sophia Antipolis,

France.E-mail:enrico.zio@mines-paristech.fr

Eminent Scholar at the Department of Nuclear Engineering, Kyung Hee University, Seoul, South Korea.

\begin{abstract}
Bearings are one of the most used components in rotating machinery. They are also the components which suffer from the damage most frequently. For this reason, accurate prediction of the remaining useful life (RUL) of this equipment is very important. In this paper, a Bayesian approach is presented for the RUL prediction of rolling bearings, based on a two-stage processing of the degradation feature of vibration data collected experimentally. Threshold level crossing of the vibration signal in time is as a defect detection indicator. Then, a modified Paris crack growth model is developed for the bearing's defect propagation on slow-time and fast-time scales. Probability distributions of the uncertain parameters in the model are introduced and the Metropolis-Hasting algorithm is applied to the Markov chain Monte Carlo method for generating samples from the posterior distribution, which are used to estimate the RUL distribution and to describe different types of bearing degradation and fault growth processes.
\end{abstract}

Keywords: Remaining useful life, Rolling bearing, Vibration, Uncertainty, Bayesian approach, Metropolis-Hasting algorithm.

\section{Introduction}

According to the International Standard Organization, failure prognostic corresponds to the "analysis of the various fault symptoms and failure growth to predict future condition and residual life within design parameters" (ISO 13381-1:2015).

Three categories of methods have been developed to estimate the remaining useful life (RUL) of rolling bearings: physical model-based methods, data-driven methods and combined methods. The advantage of physical model-based methods is that they are more general and long-term predictable, but they suffer from the complexity of the degradation phenomena, so that most of the times it is difficult to develop the models in practice. On the other hand, data-driven methods are easier to use if sufficient data are available for training them. Combined methods use both of the methods to take advantage of their positive characteristics (Zio (2013)).

Several physical models have been developed, such as Paris crack growth model (Li et al. (1999)), Forman growth model (Shih et al. (1997)), Spall progression model (Choi et al. (2007)), and Huang growth model (Huang et al. 2008). They describe the evolution of physical states and structural damage levels according to the mechanistic laws that relate to the material response of bearings depending on the loading conditions. Some researchers have used Paris crack growth model for the RUL prediction of bearings. Li et al. (1999) proposed an adaptive method, which uses the vibration level for fault intensity estimation and, then, predicts the degradation rate based on the Paris law.

Zio et al. (2011) introduced a particle filteringbased prognostic framework for nonlinear components. They applied it on the modified Paris

Proceedings of the 30th European Safety and Reliability Conference and

the 15th Probabilistic Safety Assessment and Management Conference

Edited by Piero Baraldi, Francesco Di Maio and Enrico Zio

Copyright (C) ESREL2020-PSAM15 Organizers.Published by Research Publishing, Singapore.

ISBN: 978-981-14-8593-0; doi:10.3850/978-981-14-8593-0 
crack model for non-destructive ultrasonic inspections.

Li et al. (2014) presented a framework for the RUL prediction of rolling bearings by using particle filtering and a new virtual health indicator extracted from multiple features. They transformed Paris crack model to a state-space equation, in which the extracted indicator is put and, finally, the model parameters are updated by using a particle filtering. This particle filteringbased approach was shown to perform better than the ANFIS-based approach.

Qian et al. (2017) proposed a multi-time space approach by using a modified Paris model for fault growth. The phase space warping method is employed for extracting a new feature. Also, the relationship between this feature and crack growth has been studied and used for the RUL prediction of rolling bearings based on the physical model.

Behzad et al. (2017) employed the level crossing of the time signal as an indicator for fault size estimation. They extracted a new model for a twostage process based on the Paris law equation and studied the effect of the transition point from the slow stage to the fast stage by considering a normal distribution for the RUL prediction.

However, the values of the parameters in the physical model are uncertain. Therefore, probability distributions must be introduced to represent this uncertainty and can give a more realistic prediction. In this paper, a new modified Paris law equation is developed for a two-stage degradation process. Then, probability distributions are introduced for the uncertain parameters. The Bayesian method is, then, used to update the model parameters based on the measured data. Finally, the bearing RUL is estimated.

\section{Model Definition}

Paris formula describes the relationship between the crack growth rate and the defect conditions $(\mathrm{Li}$ et al. (1999)):

$$
\frac{d l}{d N}=C_{0} \Delta k^{n}
$$

where $l$ is the crack growth, $N$ is the time (e.g. loading cycle), $C_{0}$ and $n$ are material constants and $\Delta k$ is a stress intensity factor that can be estimated in the rolling bearings as $(\mathrm{Xu}$ et al. (2012)):

$$
\Delta k=\tau_{\max } \sqrt{\pi l} Y
$$

where $\tau_{\max }$ is the maximum shear stress and $Y$ is a geometric correction factor that depends on the crack shape.

By substituting $\Delta k$ in Eq. (1) and integrating, the Paris equation can be written as:

$$
\int d N=\int \frac{d l}{C_{0}\left(\tau_{\max } \sqrt{\pi l} Y\right)^{n}}
$$

where $n=2$ is considered for commonly used rolling bearing materials (Qian et al. (2017)):

$$
\int d N=\int \frac{d l}{C_{0}\left(\tau_{\max } \sqrt{\pi l} Y\right)^{2}}
$$

In practice, the damage level of machinery, such as the crack lengths and the wear areas, is not directly observable. Therefore, extracting the suitable and sensitive health features of machinery is required in the fault diagnosis and prediction. Behzad et al. (2009) have shown experimentally that the level crossing rate $(z)$ of the time wave signal at threshold level has a linear relationship with defect size $(l)$. Threshold level is considered $2 \sigma_{0}$ (The standard deviation of acceleration signal for normal stage) for rolling bearings (Behzad et al. (2017)). By using this indicator in Eq. (4), Paris law equation becomes:

$$
\begin{gathered}
l=\alpha z \\
\int d N=\int \frac{d z}{\sqrt{\pi} C_{0} \tau_{\max }^{2} z Y^{2}}
\end{gathered}
$$

In Eq. (5), parameter $D$ is introduced as:

$$
D=\frac{1}{\sqrt{\pi} C_{0} \tau_{\max }{ }^{2} Y^{2}}
$$

Therefore, Eq. (6) can be reformulated as:

$$
\int d N=D \int \frac{d z}{z}
$$

By integrating Eq. (7), the level crossing indicator can be calculated:

$$
N+\alpha=D \ln z \quad z=e \frac{N+\alpha}{D} \quad N=t,
$$




$$
\begin{gathered}
C=e^{\frac{\alpha}{D}} \beta=\frac{1}{D} \\
z=C e^{\beta t}
\end{gathered}
$$

where the parameters $C$ and $\beta$ are uncertain and need to be estimated from historical data.

In the initial crack growth stage, the crack growth rate is so slow that the variation of the indicator is not very significant. Only after the crack initiation stage, crack propagation will enter a stage where crack growth is accelerated due to the material fatigue caused by repetitive loading as the bearing operates.

The model for two-stage degradation can be represented as follows:

$$
z=\left\{\begin{array}{lc}
C_{1} e^{\beta_{1}\left(t-t_{\text {initial }}\right)} & t \leq t_{\text {tra }} \\
C_{2} e^{\beta_{2}\left(t-t_{\text {tra }}\right)} & t>t_{\text {tra }}
\end{array}\right.
$$

where the parameters $C_{1}, C_{2}, t_{\text {tra }}, \beta_{1}, \beta_{2}, t_{\text {initial }}$ are uncertain and need to be estimated from historical data.

\section{Prediction updating using the Bayesian method}

Bayesian inference can be used for updating. In the form of probability density function (PDF), it can be expressed as (Dong et al. (2019)):

$$
f_{\theta}(\theta \mid Y=y)=\frac{f_{Y}(y \mid \theta=\theta) f_{\theta}(\theta)}{f_{Y}(y)}
$$

- $f_{\theta}(\theta) \rightarrow$ joint PDF of the uncertain parameter $\theta$

- $\quad f_{Y}(y) \rightarrow$ normalized constant

- $f_{Y}(y \mid \theta=\theta) \rightarrow$ likelihood function of evidence y given the parameter value $\theta$

- $f_{\theta}(\theta \mid Y=y) \rightarrow$ posterior PDF of the uncertain parameter $\theta$.

Assuming that there are $N_{\text {data }}$ measurements available, Bayes' Theorem is applied as:

$$
f_{\theta}(\theta \mid Y=y)=\frac{1}{Q} \prod_{i=1}^{N_{\text {data }}}\left[f_{Y}\left(y_{i} \mid \theta=\theta\right)\right] f_{\theta}(\theta)
$$

In practice, the measured degradation data $\left(y_{i}, t_{i}\right)$ $i=1,2 \ldots, N_{\text {data }}$ are collected at discrete times, so the degradation model is also evaluated at the same discrete times, as $\tilde{y}_{i}(\theta)=\tilde{y}_{i}\left(\theta, t_{i}\right), \quad i=$ $1,2, \ldots, N_{\text {data }}$. Then, the likelihood function of the $i_{t h}$ measured data can be defined as (Dang et al. (2019)):

$$
\begin{gathered}
f_{Y}\left(y_{i} \mid \theta=\theta\right)=\frac{1}{s \sqrt{2 \pi}} \exp \left[-\frac{1}{2 s^{2}}\left(y_{i}-\tilde{y}_{i}\right)^{2}\right], \\
i=1,2, \ldots . N
\end{gathered}
$$

By substituting the likelihood function in Eq. (11), the posterior joint PDF can be calculated by multiplying all likelihood functions and the prior PDF as:

$$
\begin{gathered}
f_{\theta}(\theta \mid y)=\frac{1}{Q S^{N_{\text {data }}}} \times \\
\exp \left[-\frac{1}{2 s^{2}} \sum_{i=1}^{N_{\text {data }}}\left(y_{i}-\tilde{y}_{i}\right)^{2}\right] f_{\theta}(\theta)
\end{gathered}
$$

The prior distribution for the uncertain parameters is estimated based on historical 'run-to-failure' data. Then, a sufficient number of random samples is drawn using the Markov chain Monte Carlo (MCMC) method. The MCMC method generates random samples using the Markov chain process, in which the distribution of the next sample depends only on the current sample (Marseguerra et al. (2002)). The MetropolisHasting algorithm is used to accept or reject the data generated with the MCMC method.

\section{Experimental test and results}

To study the performance of the proposed framework based on the Bayesian method and the extended Paris law equation, experimental vibration data collected by NSF I/UCR center are considered (Lee et al. (2007)). The experimental setup is shown in Fig.1. Four rolling bearings are installed on a shaft and operated under constant conditions $(2000 \mathrm{rpm}$ speed and $6000 \mathrm{lb}$ radial load). The data set collected contains three run-tofailure experiments for four of the ball bearings. The sampling frequency is $20 \mathrm{kHz}$, the length of the data is 2156 and the recording is repeated every ten seconds. where $Q$ is the product of all marginal PDFs and is a normalizing constant factor. 

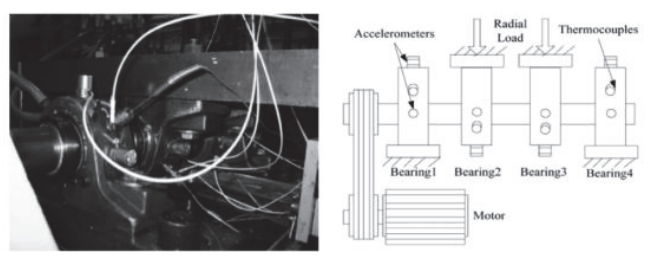

Fig. 1. Experimental setup for bearing run-to-failure test

The acceleration data of the third bearing in the first experiment has a two-stage degradation pattern. For this reason, this experiment is considered for the investigation of the proposed method. Fig. 1 and Fig. 2 show the trend of the RMS and level crossing from the healthy stage to the end of life.

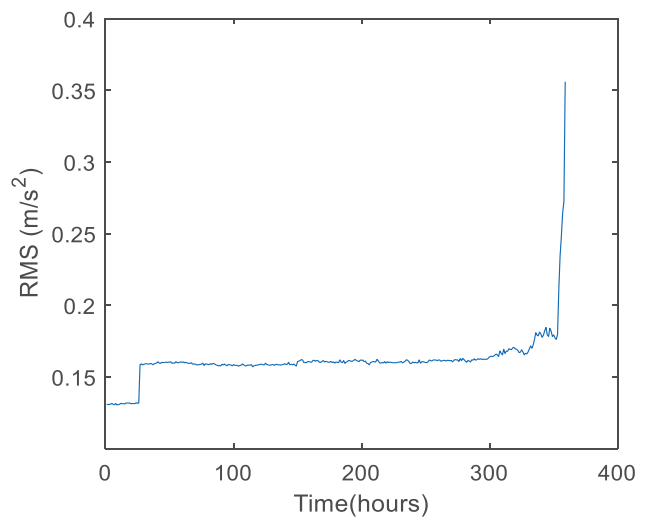

Fig.2. RMS trend in the rolling bearing life

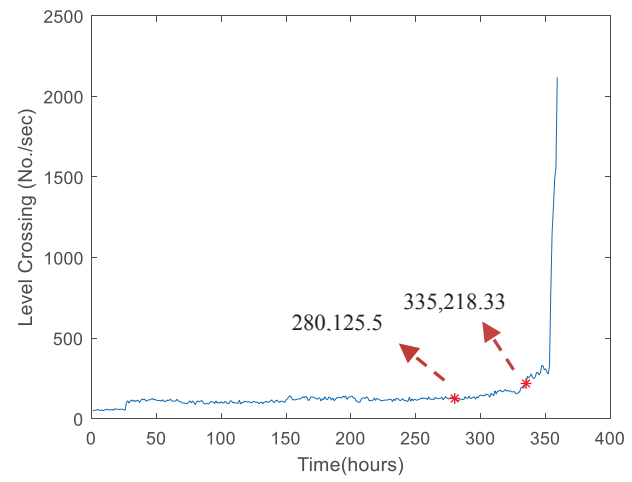

Fig.3. Level crossing trend in the rolling bearing life

In Fig. 2 and Fig. 3, the degradation indicator of the test bearing involves three distinct stages, the normal, the failure propagation and the severe failure stage. In the normal stage, fluctuations are within a very small range, which means that the bearing is operating under normal conditions during this time. Then, the level crossing and RMS increase gradually. Thus, defects are found in the bearing, which propagate for a long time before the last stage. At the final stage, a sudden fluctuation occurs and the bearing degrades to failure rapidly, in a short time. For the RUL prediction based on the proposed method, the failure propagation and severe failure stages are considered.

For numerical simulation, the threshold of the level crossing indicator is set to $400(\mathrm{No} / \mathrm{sec})$ based on experimental tests and also comparing with the RMS trend. Estimated values of the uncertain parameters of the model, $C_{1}, C_{2}, \beta_{1}$ and $\beta_{2}$ are determined using the nonlinear least square method. The results for the start and end of the slow stage of degradation are presented in Table 1.

Table 1. Parameters for degradation simulation

\begin{tabular}{cl}
\hline$t_{\text {initial }}$ & $280 \mathrm{hr}$ \\
$t_{\text {tra }}$ & $335 \mathrm{hr}$ \\
$C_{1 \_ \text {inital }}$ & 118.58 \\
$C_{2 \_ \text {inital }}$ & 60 \\
$\beta_{1 \text { _inital }}$ & 0.0113 \\
$\beta_{2 \_ \text {initial }}$ & 0.02 \\
$C_{1}$ & {$[100130]$} \\
$C_{2}$ & {$[4080]$} \\
$\beta_{1}$ & {$[0.0010 .2]$} \\
$\beta_{2}$ & {$[0.0010 .1]$} \\
\hline
\end{tabular}

The initial degradation development stage is considered from $280 \mathrm{hr}$ to $335 \mathrm{hr}$. The fast degradation propagation stage is started from 335 $\mathrm{hr}$ and continues to the end of life. The MCMC method has been performed on the basis of $N=5000$ particles for each cycle. Fig. 4 and Fig. 5 show the predicted degradation growth curve of the level crossing indicator, with confidence intervals considering one-stage and two-stage analysis. The blue lines are the real data, the red lines are the median prediction and the purple lines are $95 \%$ confidence intervals. Both of the considered methods, single-stage and doublestage, show good estimation at the slow stage and the median prediction is close to real data. At the fast stage, the predicted indicator in the two-stage solution is better than the one-stage solution. For 
convenience of comparison, the relative error of the predicted RUL with respect to the actual data at time $290 \mathrm{hr}$ is presented in Table 2. According to the results, it can be concluded that the twostage degradation prediction model can obtain good RUL prediction.

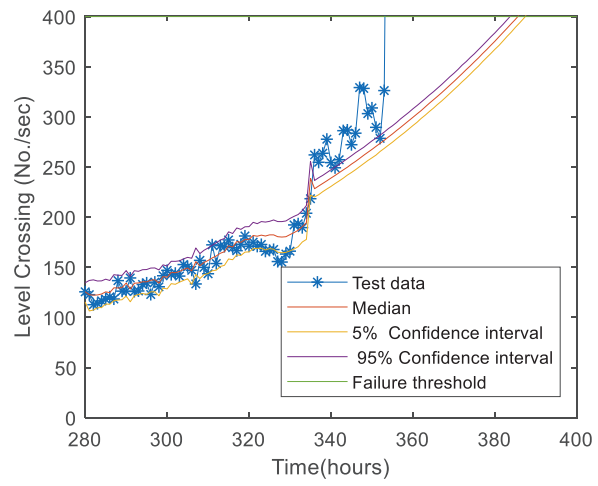

Fig. 4. Prediction results of test bearing with one-stage degradation process model

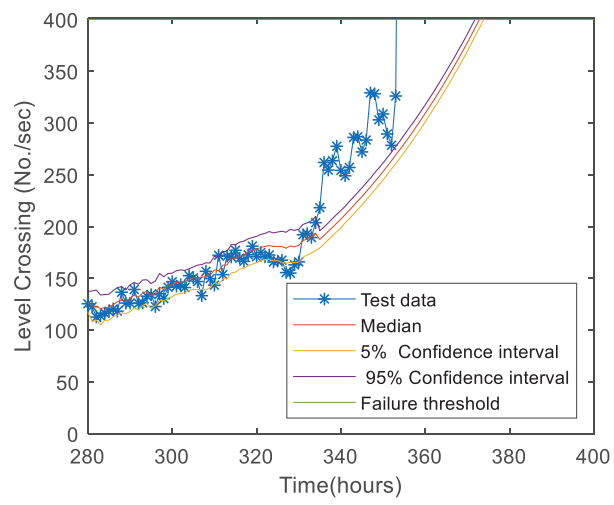

Fig. 5. Prediction results of test bearing with two-stage degradation process model
Table 2. Relative error between the predicted RUL and the real life at time 290, with three confidence intervals

\begin{tabular}{|c|c|c|}
\hline $\begin{array}{l}\text { Degradation } \\
\text { stages }\end{array}$ & $\begin{array}{c}\text { Confidence } \\
\text { interval }\end{array}$ & $\begin{array}{c}\text { Error } \\
\%\end{array}$ \\
\hline \multirow{3}{*}{ One-stage } & Median & $50 \%$ \\
\hline & $5 \%$ & $53 \%$ \\
\hline & $95 \%$ & $47 \%$ \\
\hline \multirow{3}{*}{ Two-stage } & Median & $30 \%$ \\
\hline & $5 \%$ & $31.7 \%$ \\
\hline & $95 \%$ & 28.5 \\
\hline
\end{tabular}

\section{Conclusion}

Rolling bearings are essential parts of various industrial rotating machines. Most of the time, their degradation trend is nonmonotonic and changes abruptly. Few studies have analysed different types of degradation for the prediction of the remaining useful life (RUL) of rolling bearings. The main contribution of this work is to present a framework for the prediction of the RUL of rolling bearings based on a two-stage degradation process. A Bayesian approach is adopted to consider uncertainty in the model parameters. The fast-stage results indicate that the proposed framework is able to estimate the degradation process more precisely than the onestage process. 


\section{References}

Behzad, M., A. AlandiHallaj, A. Rohani Bastami, D. Mba, B. Eftekharnejad, and B. Charnley. 2009. "Defect Size Estimation in Rolling Element Bearings Using Vibration Time Waveform.',

Www.Ingentaconnect.Com. August 1, 2009.

Behzad, Mehdi, Hesam Addin Arghan, Abbas Rohani Bastami, and Ming J. Zuo. 2017. "Prognostics of Rolling Element Bearings with the Combination of Paris Law and Reliability Method.” IEEE Xplore. July $1,2017$.

https://doi.org/10.1109/PHM.2017.8079187.

Choi, Youngsik, and C. Richard Liu. 2007. "Spall Progression Life Model for Rolling Contact Verified by Finish Hard Machined Surfaces." Wear 262 (1): 24-35. https://doi.org/10.1016/j.wear.2006.03.041.

Dong, Ting, Dawn An, and Nam H. Kim. 2019. "Prognostics 102: Efficient Bayesian-Based Prognostics Algorithm in MATLAB." Fault Detection, Diagnosis and Prognosis, February.

https://doi.org/10.5772/intechopen.82781.

Huang, Xiaoping, Moan Torgeir, and Weicheng Cui. 2008. "An Engineering Model of Fatigue Crack Growth under Variable Amplitude Loading." International Journal of Fatigue 30 (1): 210. https://doi.org/10.1016/j.ijfatigue.2007.03.004.

14:00-17:00. n.d. "ISO 13381-1:2015." ISO. https://www.iso.org/standard/51436.html.

Lee, J., Qiu, H., Yu, G., \& Lin, J. (2007). 'Rexnord technical services. Bearing Data Set', IMS, University of Cincinnati, NASA Ames Prognostics Data Repository.

https:/ti.arc.nasa.gov/tech/dash/groups/pcoe/prognosti c-data repository/.

Marseguerra, Marzio, and Enrico Zio. 2002. "Basics of the Monte Carlo Method with Application to System Reliability.’Re.Public.Polimi.It.

https://re.public.polimi.it/handle/11311/556913\#.XxR yoigzaUk

Naipeng Li, Yaguo Lei, Zongyao Liu, and Jing Lin. 2014. "A Particle Filtering-Based Approach for Remaining Useful Life Predication of Rolling Element Bearings.” IEEE Xplore. June 1, 2014.

https://doi.org/10.1109/ICPHM.2014.7036367.
Li, Y., S. Billington, C. Zhang, T. Kurfess, S. Danyluk, and S. Liang. 1999. "Adaptive Prognostics for Rolling Element Bearing Condition.” Mechanical Systems and Signal Processing 13 (1): 103-113.

https://doi.org/10.1006/mssp.1998.0183.

Qian, Yuning, Ruqiang Yan, and Robert X. Gao. 2017. "A Multi-Time Scale Approach to Remaining Useful Life Prediction in Rolling Bearing." Mechanical Systems and Signal Processing 83 (January): 549-567. https://doi.org/10.1016/j.ymssp.2016.06.031.

Shih, Yan-Shin, and Jien-Jong Chen. 1997. "Analysis of Fatigue Crack Growth on a Cracked Shaft." International Journal of Fatigue 6 (19): 477 485 .

https://www.infona.pl/resource/bwmeta1.element.else vier-a138289a-5998-3bb5-bb8c-557fflac9993.

$\mathrm{Xu}$, Dong, Jin'e Huang, Qin Zhu, Xun Chen, Yongcheng $\mathrm{Xu}$, and Shuang Wang. 2012. "Residual Fatigue Life Prediction of Ball Bearings Based on Paris Law and RMS." Chinese Journal of Mechanical Engineering 25 (2): 320-27.

https://doi.org/10.3901/cime.2012.02.320.

Zio, E. 2013. "Prognostics and Health Management of Industrial Equipment." Diagnostics and Prognostics of Engineering Systems: Methods and Techniques. 2013. https://www.igi-global.com/chapter/content/69686.

Zio, Enrico, and Giovanni Peloni. 2011. "Particle Filtering Prognostic Estimation of the Remaining Useful Life of Nonlinear Components." Reliability Engineering \& System Safety 96 (3): 403-9. https://doi.org/10.1016/i.ress.2010.08.009. 